\title{
Pain assessment in cats undergoing ovariohysterectomy by midline or lateral celiotomy through use of a previously validated multidimensional composite pain scale ${ }^{1}$
}

\author{
Jéssica Pecene Oliveira ${ }^{\mathrm{I}}$, Rodrigo Mencalha ${ }^{\mathrm{II}}$, Carlos Augusto dos Santos Sousa ${ }^{\mathrm{III}}$, Marcelo Abidu-Figueiredo ${ }^{\mathrm{IV}}$, Síria da Fonseca Jorge ${ }^{\mathrm{V}}$ \\ DOI: http://dx.doi.org/10.1590/S0102-8650201400160002 \\ ${ }^{\mathrm{I}}$ Graduate student, School of Veterinary Medicine, Serra dos Orgaos Educational Foundation (CEUA-UNIFESO), Teresopolis-RJ, Brazil. Technical \\ procedures, acquisition of data. \\ IIMSc, Assistant Professor, Department of Veterinary Surgery and Anesthesiology, School of Veterinary Medicine, Valenca Higher Education Center \\ (CESVA), Valenca-RJ, Brazil. Intellectual and scientific content of the study, statistical analysis, manuscript writing, critical revision. \\ IIIFellow Master degree, Postgraduate Program in Animal Biology, School of Biology, Animal Anatomy Area, Federal Rural University of Rio de \\ Janeiro (UFRRJ), Seropedica-RJ, Brazil. Technical procedures, acquisition and interpretation of data. \\ ${ }^{\text {IV }} \mathrm{PhD}$, Associate Professor, Department of Animal Biology, Anatomy Area, School of Veterinary Medicine, UFRRJ, Seropedica-RJ, Brazil. Intellectual \\ and scientific content of the study, statistical analysis, critical revision. \\ ${ }^{v}$ MSc, Assistant Professor, Department of Veterinary Clinic and Surgery, School of Veterinary Medicine, UNIFESO, Teresopolis-RJ, Brazil. Intellectual \\ and scientific content of the study, statistical analysis, technical procedures, critical revision.
}

\section{ABSTRACT}

PURPOSE: To assess pain in the immediate postoperative period in cats submitted into two different celiotomy techniques for ovariohysterectomy.

METHODS: Fourteen healthy female cats up to three years old with a mean weight $2.75 \mathrm{~kg}$, without breed specification, were used in this double blind experiment. The animals were randomly assigned to two treatments: I- ovariohysterectomy by lateral approach (LA) or II - by midline approach (MA). The anesthesia consisted of acepromazine $\left(0.1 \mathrm{mg} \cdot \mathrm{kg}^{-1}\right)$ and midazolam $\left(0.25 \mathrm{mg} \cdot \mathrm{kg}^{-1}\right)$ followed isoflurane vaporization to induce and maintain hypnosis. A bolus of fentanyl $\left(5 \mu \mathrm{g} \cdot \mathrm{kg}^{-1}\right)$ was administered intravenously to provide intraoperative analgesia. After surgery, pain scores were assessed through a multidimensional composite pain scale at four different times.

RESULTS: Generally all factors related to psychomotor changes and pain expression showed higher scores in cats neutered by LA, but only psychomotor changes and total pain score presented statistical differences $(\mathrm{p}<0.05)$. The animals that underwent lateral celiotomy showed higher pain scores, at 1, 4 and 6 hours after surgery.

CONCLUSIONS: Multidimensional analgesic scales were highly reliable. There was a tendency for the cats neutered by lateral approach to suffer more postoperative pain, including requiring a large number of analgesic rescues.

Key words: Laparotomy. Hysterectomy. Pain Measurement. Scales. Cats. 


\section{Introduction}

Ovariohysterectomy $(\mathrm{OH})$ is one of the most common surgeries in veterinary medicine. Besides reproductive control, this surgical procedure is indicated in cases of pyometra, metritis, dystocia, mammary tumors and reproductive disorders. In cats, besides the indications described, signs of oestrus such as increased vocalization, rolling on the ground and a very short interoestrous interval can negatively influence the relationship between cat and owner, and prompt the owner to seek a method of reproduction control $^{1}$

Due to media campaigns, society is becoming more participatory regarding population control of domestic animals, causing a steady increase in surgical castrations in recent years. Beyond control of zoonoses, population control improves animal welfare ${ }^{2-3}$.

Surgical trauma is associated with activation of the sympathetic-adrenal axis, with consequent breakdown of homeostasis and increased recovery time. By minimizing postoperative pain, veterinary surgeons provide better results ${ }^{4}$. Thus, less invasive techniques are being incorporated into the veterinary routine to enable better surgical recovery ${ }^{5}$.

To perform celiotomy for $\mathrm{OH}$, the lateral approach is an alternative to the traditional approach (ventral midline). This alternative is not conventional in small animals, but is often an alternative for farm animals and pet reptiles. However, even though not common in treating pets, flank castration is technically possible to be held and considered easy to perform by trained professionals ${ }^{6-7}$.

Even today, perioperative and postoperative pain is neglected in several animal species ${ }^{8-9}$, a fact closely related to the difficulty of recognition, management and treatment of animal pain. For example, the protocols established in $\mathrm{OH}$ at some locations are devoid of analgesics or carried out in sub-therapeutic doses, in disagreement with the principles of ethics and animal welfare.

Due to the difficulty of identifying pain in animals, the postoperative treatment can often be inappropriate ${ }^{10-11}$. Thus, the development of more accurate methods of evaluation is crucial for optimal management of pain. Despite the availability of numerous pain measurement scales for small animals, the only aspects that have been validated are responsiveness and reliability. Responsiveness assesses the ability of the instruments (scales) to detect significant changes in pain intensity over time or in response to clinical intervention to relieve pain (analgesics) ${ }^{12}$.

Validated scales for the assessment of acute pain in dogs are available in the literature ${ }^{13-15}$, but in relation to cats there is serious shortage of these instruments. A multidimensional scale for assessment of acute postoperative pain was initially developed to evaluate analgesic response in cats undergoing $\mathrm{OH}$, and has been carefully refined ${ }^{16-18}$.

The aim of this study was to evaluate postoperative pain in cats undergoing lateral or midline celiotomy to perform ovariohysterectomy, through the Botucatu multidimensional composite pain scale.

\section{Methods}

Research was approved by the Ethics Committee of Serra dos Orgaos Educational Foundation (CEUA - UNIFESO) under license number $0377 / 13$ and the surgeries were performed at the Clinical School of Undergraduate Veterinary Medicine.

Fourteen healthy female cats up to three years old, with body weight (mean \pm SD) of $2.75 \pm 0.85 \mathrm{~kg}$, without breed specification, were used in this experiment. The Stray Animal Center of Teresopolis, Rio de Janeiro, provided the animals for this study.

The animals were randomly assigned to two groups: I- (Lateral Approach - LA) ovariohysterectomy by lateral (flank) celiotomy $(\mathrm{n}=7)$; and II- (Midline Approach - MA); ovariohysterectomy by midline celiotomy $(n=7)$. The flank and the abdomen region of all cats were shaved and two different sutures on each cat were made: one suture in the surgical wound (midline or flank) and another "fictitious" suture in one of the above (surgical access opposite), in order to minimize the influence of this knowledge on the judgment of the evaluator (double blind trial).

The cats were subjected to basic protocols inherent in pre-anesthetic evaluation to determine their physical condition and surgical risk. Basic laboratory tests, measurement of temperature, respiratory and cardiac auscultation and analysis of body score were performed. After completion of fasting and an water deprivation, the cats were pre-medicated with acepromazine $\left(0.1 \mathrm{mg} / \mathrm{kg}^{-} 1\right.$, Vetnil Indústria e Comércio de Produtos Veterinários Ltda., Louveira, São Paulo, Brazil) and midazolam $\left(0.25 \mathrm{mg} / \mathrm{kg}^{-1}\right.$, Roche, São Paulo, Brazil), both administered intramuscularly, anesthesia induced and maintenance through vaporization of isoflurane (Cristália, São Paulo, Brazil). A bolus of fentanyl $\left(5 \mu \mathrm{g} . \mathrm{kg}^{-1}\right)$ was administered intravenously to provide intraoperative analgesia.

The same surgeon operated on all animals. The LA group underwent lateral celiotomy in the right flank and MA group underwent midline celiotomy.

The midline celiotomy was performed through a retroumbilical incision in the skin, subcutaneous cellular tissue and linea alba to access the abdominal cavity. The right and left ovaries were exposed, their vascular pedicles ligated with a longterm absorbable surgical thread (polyglycolic acid 2.0, São Luis de 
Montes Belos, Goiânia, Brazil) and later removed along with the uterus and cervix. The abdominal wall was continuously sutured, anchored with the same thread and then a simple discontinuous suture (" $x$ ") on the skin was performed with a nonabsorbable surgical thread (nylon monofilament 2-0, São Luis de Montes Belos, Goiânia, Brazil)

For lateral celiotomy, an incision was made on the skin of the right flank, in the middle third between the last rib and the iliac crest, across the midline. The musculature was divulsed in the direction of the fibers and the peritoneum incised. The ovaries, uterine horns and the uterine body were removed the same way as in the midline access. The musculature was continuously and individually sutured (external oblique, internally transverse in the abdomen), anchored with long-lasting absorbable surgical thread (polyglycolic acid 2.0, São Luis de Montes Belos, Goiânia, Brazil) and then a single discontinuous suture (" $\mathrm{x}$ ") was performed in the skin with a nonabsorbable surgical thread (nylon monofilament 2-0, São Luis de Montes Belos, Goiânia, Brazil)

After surgery, the cats were kept in individual stainless steel cages measuring $60 \mathrm{~cm} \mathrm{X} 60 \mathrm{~cm}$, with a single bed for the safe and comfortable conduction of analgesic evaluations.

The cats were subjected to pain evaluation at four different moments: M1 (one hour after surgery), M2 (two hours after surgery), M3 (four hours after surgery) and M4 (six hours after surgery). A multidimensional composite pain scale - UNESP Botucatu, previously validated $^{17}$, was used for acute postoperative pain evaluation. This scale comprises four descriptive levels, which are ordered categorically and anchored at $0,1,2$ and 3, so that " 0 " represents no change or normality and " 3 " is greatest changes. The total score is calculated from the sum of the items' scores, ranging from 0 (no change) to 30 (maximum pain). The blood pressure parameter was not considered, so that the maximum score was 27 points.

Analgesic rescue was performed at the time the score reached 8 points $(0-8=$ slight discomfort; $9-15=$ moderate discomfort; and $16-27=$ severe discomfort). The appetite evaluation was performed with a small amount of tuna flavor cat food (Whiskas - Mars Incorporated, McLean, Virginia, USA) and play evaluation with a colored tape and toy mouse.

The analgesic rescue was performed according to the need of each animal by intramuscular administration of $3 \mathrm{mg} \cdot \mathrm{kg}^{-1}$ of tramadol (tramadol chlorhydrate, Laboratório Teuto Brasileiro, Anápolis, Goiás, Brazil) and $0.1 \mathrm{mg} \cdot \mathrm{kg}^{-1}$ of $0.2 \%$ meloxicam (Ourofino Agronegócio, Cravinhos, São Paulo, Brazil). The anesthetic/analgesic protocol chosen aimed to provide adequate analgesia during and after surgery, but without interfering directly in the pain assessments by the selected techniques.
Statistical analyses were performed using Graphpad Prim for Mac OS X version 6.0. The means and standard deviations were calculated and compared in both surgical groups. The independent nonparametric data present in this study were analyzed through the Mann-Whitney test. The independent parametric data were analyzed through unpaired Student t-test. These tests were used to compare the surgical time in both techniques. For comparison of the total pain scores (psychomotor changes and pain expression) at each evaluation moment, the Kruskal-Wallis test was performed to test whether the set of samples came from the same distribution. Significant difference was accepted when $\mathrm{p}<0.05$.

\section{Results}

No statistical difference was observed in surgical time between the MA group (mean $\pm \mathrm{SD}, 24.5 \pm 4.5 \mathrm{~min}$ ) and LA group (mean $\pm \mathrm{SD}, 28.0 \pm 5.0 \mathrm{~min}$ ).

The results obtained through the UNESP-Botucatu multidimensional composite pain scale for assessing postoperative pain in cats are shown in Tables 1 and 2.

TABLE 1 - Pain score (0-27) according to the double blind observer on each item of the multidimensional table.

\begin{tabular}{lccc}
\hline Item & Approach & Mean & SD \\
\hline Posture & Midline & 0.83 & 1.32 \\
& Lateral & 1.83 & 1.16 \\
Comfort & Midline & 1.83 & 0.98 \\
& Lateral & 2.83 & 1.60 \\
Activity & Midline & 2.00 & 0.68 \\
& Lateral & 4.33 & 1.56 \\
Attitude & Midline & 2.00 & 1.26 \\
& Lateral & 3.00 & 2.28 \\
Miscellaneous behavior & Midline & 2.83 & 1.83 \\
& Lateral & 3.33 & 1.21 \\
Surgical wound palpation & Midline & 2.83 & 0.98 \\
& Lateral & 4.66 & 3.20 \\
Abdomen/flank palpation & Midline & 0.50 & 0.83 \\
& Lateral & 1.16 & 1.83 \\
Appetite & Midline & 7.50 & 2.73 \\
Vocalization & Lateral & 4.16 & 2.78 \\
Total pain score $(0-27) *$ & Midline & 0.66 & 1.63 \\
Partial pain score $(0-12)$ & Lateral & 1.50 & 1.76 \\
(psychomotor changes) $+*$ & Midline & 1.68 & 1.54 \\
Partial pain score $(0-12)$ & Lateral & 2.83 & 2.43 \\
Vain expression) ++ & Midline & 1.66 & 1.37 \\
& Lateral & 3.00 & 2.43 \\
& Midline & 1.70 & 1.73 \\
& Lateral & 2.66 & 2.46 \\
\hline
\end{tabular}

Values are shown as mean \pm standard deviation. + Psychomotor changes (posture, comfort, activity and attitude). ++ Pain expression (miscellaneous behavior, surgical wound palpation, abdomen/flank palpation and vocalization). ${ }^{*} \mathrm{p}>0.05$. 
TABLE 2 - Total multidimensional pain score (0-27) according to the double blind observer at each moment evaluated.

\begin{tabular}{cccc}
\hline Evaluation Moment & Approach & Mean & SD \\
\hline M1 & Midline & 4.33 & 2.94 \\
& Lateral & 7.16 & 5.74 \\
M2 & Midline & 6.50 & 4.32 \\
& Lateral & 7.00 & 4.69 \\
M3 & Midline & 4.83 & 3.31 \\
& Lateral & 8.33 & 3.88 \\
M4 & Midline & 5.33 & 6.93 \\
& Lateral & 4.33 & 2.42 \\
\hline
\end{tabular}

Values are shown as mean \pm standard deviation. M1 = 1 hour after surgery; M2 = 2 hours after surgery; M3 $=4$ hours after surgery; M4 $=6$ hours after surgery.

The items present in the multidimensional scale when compared individually between the experimental groups showed no significant differences, but when the total pain score and partial pain score (psychomotor changes) were compared, both showed statistically significant differences ( $\mathrm{p}=0.023$ and 0.047 , respectively - Table 1 ).

According to the subdivision of the UNESP Botucatu pain scale, we assessed psychomotor aspects (posture, comfort, activity and attitude) and pain expression (miscellaneous behavior, reaction to palpation of the surgical wound, reaction to palpation of abdomen or flank, and vocalization).

Animals belonging to the MA group showed consistent changes in the posture criteria, with an arched appearance. Furthermore, the animals belonging de the LA group reaching the maximum score, indicated by excessive restlessness, with frequent changes of position.

The LA group showed greater discomfort than the MA group. Both groups had at least an indication of the maximum score. Higher frequency of score " 1 " was observed in the MA group, which indicates little responsiveness when the animal is stimulated by the observer.

The LA group appeared more reluctant to move, while the MA animals behaved more spontaneously. In this aspect, there was greater frequency of score " 2 ", mainly in LA, where the animal presents itself but still only moves slightly when stimulated.

The MA group expressed lower attitude than the LA group, with frequent aggression in cats of the LA and the more frequent disinterest in MA cats. In general, the cats were not interested much in playing, and were thus evaluated according to their response to calls and caresses.

Miscellaneous behavior was also evaluated in both groups. The LA group expressed a greater variation in behavior, with licking and/or biting at the wound region observed, while the cats from the MA group only partially closed their eyes.
The LA group showed more reaction to palpation of the wound than the MA group (Figure 1). The LA group showed maximum response to palpation when the wound was touched. In the MA group, although the most frequent score was "1", some animals scored " 2 " and " 3 ", which indicates vocalization and/or biting when the wound is touched or approached, respectively. Four cats expressed reaction to palpation in the region where there was no wounded, two in each group.

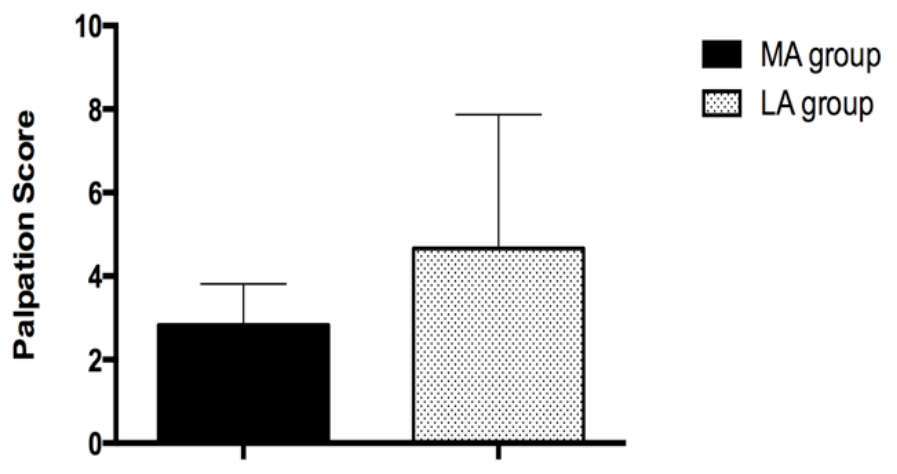

FIGURE 1 - Total palpation score in postoperative period (M1, M2, M3 and M4) between midline approach and lateral approach.

Cats submitted to the midline celiotomy had higher pain scores two hours after the surgical procedure (M2). At the other time points assessed (M1, M3, M4), the cats showed more stability in scores (Table 2 and Figure 2). While M2 had the highest scores, just one cat was assigned a score greater than 8 , with non-rescue analgesic required for the other, which had scores below 8 .

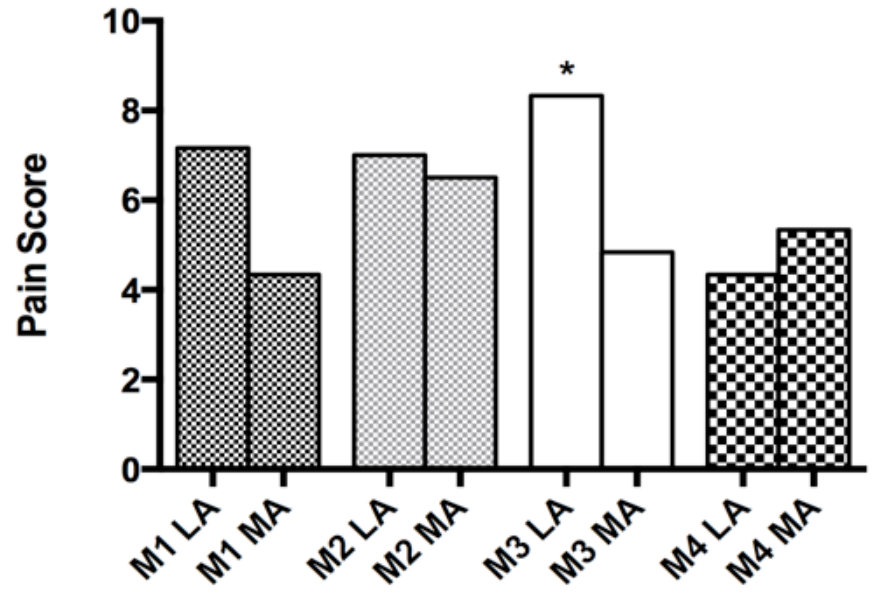

FIGURE 2 - Total pain score in postoperative period (M1, M2, M3 and M4) between midline approach (MA) and lateral approach (LA). M1 (1 hour after surgery), M2 (2 hours), M3 (4 hours) and M4 (6 hours). ${ }^{*} \mathrm{p}<0.05$.

There was greater variation between scores in the LA group, indicating clinical instability (Table 2 and Figure 2). At moment M3 (4 hours) there was an increase in the critical pain score in 4 of 6 animals evaluated. Finally, there was decreased pain 
at M4 (six hours). Statistical difference was observed between the two groups at M3 $(\mathrm{p}=0.047)$.

During the total period evaluated, three analgesic rescues in the MA group and six in the LA group were performed. This result indicates that the LA group had higher pathological pain (score $>8$ ).

There was no pattern in performing these rescues, suggesting no relationship between the emergence of pathological pain with the postoperative period (M1 - M4).

For the total pain scores, the cats that underwent flank celiotomy had higher pain at four hours after surgery (M3) than the cats submitted to the midline celiotomy. Six hours after surgery (M4), this relationship was reversed, because the LA received more analgesic rescues.

\section{Discussion}

The mean surgical time was longer in the lateral approach group ( $28 \pm 5$ versus $24.5 \pm 4.5$ minutes, but this result was not significant. This finding corroborates that of study carried out in the United Kingdom ${ }^{6}$. In that study, at a veterinary teaching hospital, 32 cats were spayed by the midline approach and 34 by the flank approach, by undergraduate students under the continuous supervision of a veterinary surgeon. The total duration of the surgery and each student's assessment of the difficulty of the surgery were not significantly different between the two groups. The time taken from the skin incision to entering the peritoneum was significantly longer with the flank approach, but finding the uterus took significantly longer with the midline approach.

In the present work the operations were performed by a single surgeon, providing greater reliability to the results. This is in agreement with the study mentioned above, in which the researchers compared the use of different surgeons versus a single surgeon and concluded that the best results were obtained with one surgeon.

We aimed to use of a multidimensional scale to evaluate postoperative pain in cats submitted to two different castration techniques. This scale, which has recently been validated ${ }^{17}$, is a major breakthrough in the evaluation of acute postoperative pain in cats by minimizing the subjectivity factor ${ }^{16,18}$. Previously, onedimensional or adapted scales from other species were the only tools for assessing pain in cats. The scale used in this paper (English version of the UNESP-Botucatu-MCPS) is a valid, reliable, responsive scale for assessing acute pain in cats undergoing $\mathrm{OHE}$, when used by anesthesiologists and anesthesia technicians. In our study, an experienced anesthesiologist and two undergraduates student, previously trained, participated in the assessments of pain.

MA group had less appetite than the LA group, However, this result may have been influenced by the fact that the MA group, which had less pain at first assessment (M1), ate more and were satiated at subsequent moments (M2, M3, M4). Individuals the LA tended to eat small amounts at all times evaluated. Therefore, if this criterion is assessed in isolation, it can lead to erroneous conclusions and when considered together it can increase or decrease the final score of the table, leading to unnecessary rescues or no rescues when they are needed. This finding contradicts those of other authors, who have mentioned activity parameters, miscellaneous behavior and attitude as evaluation terms with lower reliability ${ }^{18}$

As previously mentioned, the cats submitted to the midline celiotomy showed higher pain scores two hours after the surgical procedure, while at the other time points assessed, the cats showed stable scores. Only one cat was considered the exception, because of a progressive increase in the pain level. This can be explained by the stress of this animal. This result disagrees with the findings reported in a recent paper, in which the authors reported greater variability in the results obtained one hour after surgery ${ }^{17}$.

Regarding analgesic rescues, more were performed in the LA group. This result suggests that the LA group had higher pathological pain (above 8). Even though the optimum analgesic intervention for the proposed scale was $>8$, furthermore, in some animals, analgesia was performed with scores $<8$, according to a clinical trial. These data are in accordance with a research article that affirms the need for information to assist in clinical decision about pain therapy ${ }^{19}$. In a recent study ${ }^{17}$, the cut-off point for rescue analgesia was $>7$ (range 0-30 points), with $96.5 \%$ sensitivity and $99.5 \%$ specificity.

After adding the pain thresholds measured after each time interval, we calculated the mean scores, which showed that the cats that underwent flank celiotomy had a higher upper pain threshold four hours after surgery (M3) than the animals submitted to midline celiotomy. These data agree with the findings of a previous study, where the authors reported higher pain scores in animals submitted to flank ovariohysterectomy ${ }^{20}$.

\section{Conclusions}

Previously validated multidimensional analgesic scales were shown to be highly reliable and responsive instruments to assess acute pain in cats after ovariohysterectomy. However, experienced and trained professionals must carefully evaluate some parameters present in these scales to minimize subjectivity. There was a tendency for the cats neutered by the flank approach to experience more postoperative pain. There was also a greater need for analgesic rescues in these animals, indicting the importance of the cutoff point for analgesic rescue. 


\section{References}

1. Goericke-Pesch S. Reproduction control in cats: new developments in non-surgical methods. J Feline Med Surg. 2010 Jul;12(7):539-46. doi: 10.1016/j.jfms.2010.05.005.

2. Welsh CP, Gruffydd-Jones TJ, Murray Jk. The neuter status of cats at four and six months of age is strongly associated with the owners intended age of neutering. Vet Rec. 2013 Jun;172(22):578. doi: 10.1136/vr.101362.

3. Finkler H, Terkel J. The contribution of cat owners' attitudes and behaviours to the free-roaming cat overpopulation in Tel Aviv, Israel. Prev Vet Med. 2012 Apr;104(1-2):125-35. doi: 10.1016/j. prevetmed.2011.11.006.

4. Holte K, Kehlet H. Epidural anaesthesia and analgesia - effects on surgival stress responses and implications for postoperative nutrition. Clin Nutr. 2002 Jun;21(3):199-206. PMID: 12127927.

5. Mayhew P. Developing minimally invasive surgery in companion animals. Vet Rec. 2011 Aug;169(7):177-8. doi: 10.1136/vr.d5125.

6. Coe RJ, Grint NJ, Tivers MS, Moore AH, Holt PE. Comparison of flank and midline approaches to the ovariohysterectomy of cats. Vet Rec. 2006 Sep;159(10):309-13. doi:10.1136/vr.159.10.309.

7. Reece CP, Nimesh MK, Wyllie RE, Jones AK, Dennison AW. Description and evaluation of a right flank, mini-laparotomy approach to canine ovariohysterectomy. Vet Rec. 2012 Sep;171(10):248. doi: 10.1136/vr.100907.

8. Capner CA, Lascelles BD, Waterman-Pearson AE. Current British veterinary attitudes to perioperative analgesia for dogs. Vet Rec. 1999 Jul;145(4):95-9. PMID: 10461733.

9. Yates D, Yetes J, Roberts M. Optimum age for neutering cats. Vet Rec. 2013 Jan;172(2):53-4. doi: 10.1136/vr.f147.

10. Hugonnard M, Leblond A, Keroack S, Cadore JL.; Troncy E. Attitudes and concerns of French veterinarians towards pain and analgesia in dogs and cats. Vet Anaesth Analg. 2004 Jul;31(3):15463. PMID: 15268686

11. Farnworth M, Adams NJ, Keown A, Waran N, Stafford K. Veterinary provision of analgesia for domestic cats (Felis catus) undergoing gonadectomy: a comparison of samples from New Zealand, Australia and the United Kingdom. N Z Vet J. 2013 Dec;62(3):11722. doi: 10.1080/00480169.2013.852447.

12. Von Baeyer VL, Spagrud LJ. Systematic review of observational (behavioral) measures of pain for children and adolescents aged 3 to 18 years. Pain. 2007 Jan;127(1-2):140-50. PMID: 16996689.

13. Firth AM, Haldane SL. Development of a scale to evaluate postoperative pain in dogs. J Am Vet Med Assoc. 1999 Mar;214(5):651-9. PMID: 10088012.

14. Holton L, Reid J, Scott M, Pawson P, Nolan A. A. Development of a behaviour-based scale to measure acute pain in dogs. Vet Rec. 2001 Apr;148(17):525-31. PMID: 11354645.

15. Morton CM, Reid J, Scott ME, Holton LL, Nolan AM. Application of scaling model to establish and validate an interval level pain scale for assessment of acute pain in dogs. Am J Vet Res. 2005 Dec;66(12):2154-66. PMID: 16379662.

16. Brondani JT, Luna SP, Padovani CR. Refinement and initial validation of a multidimensional composite scale for use in assessing acute postoperative pain in cats. Am J Vet Res. 2011 Feb;72(2):17483. doi: 10.2460/ajvr.72.2.174.

17. Brondani JT, Mama KR, Luna SP, Wright BD, Niyom S, Ambrosio J, Vogel PR, Padovani CR. Validation of the English version of the UNESP-Botucatu multidimensional composite pain scale for assessing postoperative pain in cats. BMC Vet Res. $2013 \mathrm{Jul} ; 9(143)$ doi: 10.1186/1746-6148-9-143.
18. Brondani JT, Luna SP, Minto BW, Santos BP, Beier SL, Matsubara LM, Padovani CR. Reliability and cutt-off point related to the analgesic intervention of a multidimensional composite scale to assess postoperative pain in cats. Arq Bras Med Vet Zootec. 2013 Feb;9:153-62. doi: org/10.1590/S0102-09352013000100024.

19. Reid J, Nolan AM, Hughes JM, Lascelles D, Pawson P, Scott EM. Development of the short-form Glasgow composite measure pain scale (CMPS-SF) and derivation of an analgesic intervention score. Anim Welf. 2007;16(S):97-104.

20. Burrow R, Wawra E, Pinchbeck G, Senior M, Dungdale A. Prospective evaluation of postoperative pain in cats undergoing ovariohysterectomy by a midline or flank approach. Vet Rec. 2006 May;158(19):657-71. PMID: 16699135.

\section{Correspondence:}

Rodrigo Mencalha

Faculdade de Medicina Veterinária de Valença

Centro de Ensino Superior de Valença

Avenida Sargento Vitor Hugo, 161

27.600-000 Valença - RJ Brasil

rmencalha@hotmail.com

Received: May 21, 2014

Review: July 22, 2014

Accepted: Aug 20, 2014

Conflict of interest: none

Financial source: none

${ }^{1}$ Research performed at Clinical School of Undergraduate Veterinary Medicine, Department of Clinic and Surgery, Serra dos Orgaos Educational Foundation (CEUA - UNIFESO), Teresopolis-RJ, Brazil. 